\title{
Hepatic paraoxonase 1 ameliorates dysfunctional high-density lipoprotein and atherosclerosis in scavenger receptor class $B$ type I deficient mice
}

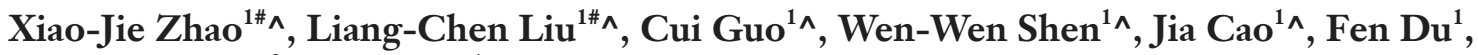 \\ Dong-Fang $\mathrm{Wu}^{2} \wedge$, Hong $\mathrm{Yu}^{1 \wedge}$
}

${ }^{1}$ Department of Biochemistry and Molecular Biology, Hubei Provincial Key Laboratory of Developmentally Originated Disease, Wuhan University School of Basic Medical Sciences, Wuhan, China; ${ }^{2}$ Department of Pharmacy, Zhongnan Hospital of Wuhan University, Wuhan, China

Contributions: (I) Conception and design: H Yu; (II) Administrative support: H Yu, DF Wu; (III) Provision of study materials or patients: J Cao, F Du; (IV) Collection and assembly data: XJ Zhao, LC Liu, C Guo, WW Shen; (V) Data analysis and interpretation: H Yu, XJ Zhao; (VI) Manuscript writing: All authors; (VII) Final approval of manuscript: All authors.

\#These authors contributed equally to this work.

Correspondence to: Hong Yu. Department of Biochemistry and Molecular Biology, Hubei Provincial Key Laboratory of Developmentally Originated Disease, Wuhan University School of Basic Medical Sciences, 185 Donghu Road, Wuhan 430071, China. Email: yu.hong@whu.edu.cn; Dong-Fang Wu. Department of Pharmacy, Zhongnan Hospital of Wuhan University, 169 Donghu Road, Wuhan 430071, China. Email: dfwu2010@whu.edu.cn.

Background: High-density lipoprotein (HDL) plays an antiatherogenic role by mediating reverse cholesterol transport (RCT), antioxidation, anti-inflammation, and endothelial cell protection. Recently, series of evidence have shown that HDL can also convert to proatherogenic HDL under certain circumstances. Plasma paraoxonase 1 (PON1) as an HDL-bound esterase, is responsible for most of the antioxidant properties of HDL. However, whether PON1 can serve as a therapeutic target of dysfunctional HDL-related atherosclerosis remains unclear.

Methods: In this study, scavenger receptor class B type I deficient $\left(S c a r b 1^{-/}\right)$mice were used as the animal model with dysfunctional HDL and increased atherosclerotic susceptibility. Hepatic PON1 overexpression and secretion into circulation were achieved by lentivirus injection through the tail vein. We monitored plasma lipids levels and lipoprotein profiles in $S c a r b 1^{-/-}$mice, and measured the levels and activities of proteins associated with HDL function. Meanwhile, lipid deposition in the liver and atherosclerotic lesions was quantified. Hepatic genes relevant to HDL metabolism and inflammation were analyzed.

Results: The results showed the relative levels of PON1 in liver and plasma were increased by 1.1-fold and 1.6-fold, respectively, and mean plasma PON1 activity was increased by 63\%. High-level PON1 increased the antioxidative and anti-inflammatory properties, promoted HDL maturation and macrophage cholesterol efflux through increasing HDL functional proteins components apolipoprotein A1 (APOA1), apolipoprotein E (APOE), and lecithin-cholesterol acyltransferase (LCAT), while decreased inflammatory protein markers, such as serum amyloid A (SAA), apolipoprotein A4 (APOA4) and alpha 1 antitrypsin (A1AT). Furthermore, hepatic PON1 overexpression linked the effects of antioxidation and anti-inflammation with HDL metabolism regulation mainly through up-regulating liver $\mathrm{X}$ receptor alpha $(\operatorname{LXR} \alpha)$ and its downstream genes. The pleiotropic effects involved promoting HDL biogenesis by raising the level of APOA1, increasing cholesterol uptake by the liver through the APOE-low density lipoprotein receptor (LDLR) pathway, and increasing cholesterol excretion into the bile, thereby reducing hepatic steatosis and aorta atherosclerosis in Western diet-fed mice.

^ ORCID: Xiao-Jie Zhao, 0000-0002-3243-8774; Liang-Chen Liu, 0000-0002-0834-4014; Cui Guo, 0000-0003-0673-9490; Wen-Wen Shen, 0000-0001-6095-8251; Jia Cao, 0000-0002-6857-0734; Dong-Fang Wu, 0000-0001-9335-4630; Hong Yu, 0000-0002-8397-7081. 


\begin{abstract}
Conclusions: Our study reveals that high-level PON1 improved dysfunctional HDL and alleviated the development of atherosclerosis in Scarb1 $1^{-1-}$ mice. It is suggested that PON1 represents a promising target of HDL-based therapeutic strategy for HDL-related atherosclerotic cardiovascular disease.
\end{abstract}

Keywords: Paraoxonase 1; high-density lipoprotein (HDL); inflammation; scavenger receptor class B type I; atherosclerosis

Submitted Feb 09, 2021. Accepted for publication Apr 23, 2021.

doi: 10.21037/atm-21-682

View this article at: https://dx.doi.org/10.21037/atm-21-682

\section{Introduction}

High-density lipoprotein (HDL) is well established as a protective factor against atherosclerosis with various functions, such as mediating reverse cholesterol transport (RCT), antioxidation, anti-inflammation, and endothelial protective function (1). HDL cholesterol (HDL-C) is still generally considered as "good cholesterol". However, recent pharmacological approaches to raise HDL-C concentration do not show reductions in cardiovascular outcomes (2). In the mid-1990s, scientists first proposed the concept of HDL dysfunction (3). Systemic and vascular inflammation has been proposed to be a trigger for converting HDL into a dysfunctional form with an atherogenic effect. Meanwhile, the lipid and protein components in the HDL particles are susceptible to oxidative modification by a variety of oxidants, such as peroxyl, aldehydes, and various myeloperoxidase (MPO)-generated oxidants. Although HDL contains antioxidative proteins that inhibit low-density lipoprotein (LDL) oxidation, oxidative modification of HDL itself is also an important cause of the functional loss of HDL. The loss of antioxidant and anti-inflammatory proteins, perhaps combined with the gain of pro-inflammatory proteins, is thought to be a key form in rendering dysfunctional HDL. Therefore, improvement of the antioxidative activity and anti-inflammatory function of HDL may be a potential strategy for HDL-targeting therapy in atherosclerotic cardiovascular disease $(4,5)$.

The growing studies have attempted to improve HDL metabolism and related functions by using HDL-targeted active proteins, such as recombinant apolipoprotein A1 (APOA1) and apolipoprotein E (APOE) mimetics, but their effectiveness for the treatment of cardiovascular disease is currently ongoing $(6,7)$. Here, we focus on a special antioxidant enzyme: paraoxonase-1 (PON1), which in the circulation exclusively associates with HDL. There is considerable evidence that PON1 as a calcium-dependent esterase, is capable of hydrolyzing various substrates such as lactones, arylesters and organophosphorus ester $(8,9)$. Especially, the hydrolytic activities of PON1 to oxidized phospholipid and hydroxides of cholesteryl linoleate in oxidative LDL (ox-LDL) have been considered as the main functions of this enzyme, which effectively protect LDL and even HDL from oxidative damage $(10,11)$. Therefore, PON1 decreases ox-LDL uptake by macrophages and stimulates macrophage cholesterol efflux, contributing to reducing the formation of foam cells (12). Meanwhile, PON1 reduces the adhesion and chemotaxis of monocytes to endothelial cells, and inhibits monocyte-to-macrophage differentiation $(13,14)$, and directly inhibits the secretion of inflammatory factors from macrophages (15). Several data in vivo have demonstrated the beneficial antioxidant and anti-inflammatory effects of PON1 against atherosclerosis. Mice lacking PON1 were observed a tendency to oxidative stress, vascular inflammation and were susceptible to atherosclerosis (16). PON1/Apoe double knockout mice exhibited increased lipoprotein oxidation and developed atherosclerotic lesions faster than Apoe knockout mice (17), while decreased atherosclerotic lesion and improved oxidation status of the aorta in PON1 transgenic mouse on Apoe deficient background (18).

Epidemiological studies have shown that the lower PON1 activity was associated with a modest inverse risk of cardiovascular disease (19). PON1 polymorphism also seems to affect atherogenesis and the occurrence of cardiovascular disease. Although the antioxidant and anti-inflammatory activity of PON1 is beneficial for the antiatherogenic ability of HDL in a mouse model of metabolic syndrome (20), whether up-regulating PON1 can improve the metabolism and function of dysfunctional HDL and play an effective atheroprotective role remains to be further studied.

The previous studies had reported that scavenger 
receptor class B type I (SR-BI) as a receptor of HDL plays a crucial role in RCT (21). Scarb1 gene deficient $\left(\right.$ Scarb1 $\left.^{-1}\right)$ mice developed by Krieger's laboratory are considered as an animal model of abnormal HDL metabolism (22), showing a high level of HDL-C accumulation in plasma, impaired RCT, reduced antioxidative and anti-inflammatory properties, and increased sensitivity to atherosclerosis (23). The increase of oxidative stress in Scarb $1^{-1}$ mice is partly due to the decreased activity of the antioxidant enzyme PON1 (24). In a previous study using lipo-proteomics analysis, we reported that decreases of APOA1 and PON1 and increases of acute-phase proteins such as serum amyloid A (SAA), apolipoprotein A4 (APOA4) and alpha 1 antitrypsin (A1AT) were biomarkers for dysfunctional HDL particles in Scarb1 ${ }^{-1-}$ mice (25). In fact, mutations in SR-BI encoding gene have recently been linked to loss-of-function of HDL and increased risk of cardiovascular disease in humans (26). Therefore, in this study, Scarb $1^{-/-}$mice fed a Western diet were used as an animal model with specific dysfunctional HDL, and the aim is to explore the effect of hepatic PON1 overexpression on dysfunctional HDL and related atherosclerosis.

We present the following article in accordance with the ARRIVE reporting checklist (available at https://dx.doi. org/10.21037/atm-21-682).

\section{Methods}

\section{Mice}

The Scarb1 heterozygous (Scarb1 ${ }^{+/}$, 1:1 mixed C57BL/6×S129 genetic background) mice were created and authorized for use by Professor Monty Krieger (Massachusetts Institute of Technology, MA, USA). Scarb1 homozygous (Scarb1 ${ }^{-1}$ ) mice were produced by hybridization between $S c a r b 1^{+/-}$ females and Scarb1 $1^{-/-}$males. Scarb1 genotypes were detected by polymerase chain reaction (PCR) of mouse tail DNA. C57BL/6 mice were purchased from Vital River Laboratory Animal Technology Company (Beijing, China). All mice were housed in microisolator cages at Wuhan University Institute of Model Animal. Animal experiments were performed under a project license (No. 2018027, 19 March 2018) granted by the Medical Animal Care and Welfare Committee for Animal Experiments of Wuhan University. All experiments were performed according to the guidelines for the care and use of laboratory animals of the Chinese Animal Welfare Committee.

\section{Generation of plasmids and production of lentivirus}

Primers used for PCR human PON1 from human liver cDNA library were forward primer, 5'-AGGTTTAAACAT GGCGAAGCTGATTGCGCTC-3', and reverse primer, 5'-TAGTTTAAACTTAGAGCTCACAGTAAAGAGC T-3'. The PCR product was digested by PmeI and cloned into a lentiviral vector $\mathrm{pWPI}$ containing tagged green fluorescent protein (GFP). The recombined vector with PON1 gene was identified by DNA sequencing. Lentivirus production was packaged as described previously (27), and the lentivirus of PON1 was expressed as Lv-PON1 and the control lentivirus as Lv-GFP.

\section{PON1 overexpression in Scarb1 $1^{-/-}$mice}

In this study, twenty female $S_{c a r b 1} 1^{-1-}$ mice at 7 months of age were randomly selected, and 10 of them were injected with Lv-GFP as the control group, while the remaining Scarb1 ${ }^{-1-}$ mice were injected with $\mathrm{Lv}-\mathrm{PON} 1$. All the mice were anesthetized and injected with lentiviruses about $1.0 \times 10^{8}$ particles via the tail vein. Two weeks after the lentiviral injection, the mice were fed with AIN76A Western diet (Beijing HFK Bioscience Co., Beijing, China) to induce atherosclerosis. The composition of the diet was described previously (27). After feeding on a Western diet for 13 weeks, the mice were euthanized, then heparin anticoagulant plasma and related tissues were collected.

\section{Plasma lipids and biochemical analysis}

The mice were anesthetized after overnight fasting, and then blood was collected via heparinized capillary tubes by retroorbital venous plexus at the specified time. The plasma was separated by centrifugation, a portion of the plasma was stored at $-80^{\circ} \mathrm{C}$. Plasma alanine aminotransferase (ALT) activity, an indicator of liver function, was determined by Mohum's method. The levels of total cholesterol (TC), free cholesterol (FC), and triglycerides (TG) in plasma were determined by enzymatic endpoint assay kits (Nanjing Jiancheng Bioengineering Institute, Nanjing, China). The measurement of plasma PON1 activity using paraoxonethyl (D9286, Sigma-Aldrich, MO, USA) as substrate was described previously (28). One unit of PON1 activity was defined as $1 \mathrm{nmol}$ of p-nitrophenol formed per minute under the definition condition and expressed as $\mathrm{U} / \mathrm{mL}$ plasma. Plasma lecithin-cholesterol acyltransferase (LCAT) 
activity was determined by LCAT COLORIMETRIC ASSAY KIT (GENMED Scientifics Inc., DE, USA). Briefly, p-nitrophenol butyrate was used as a substrate to release p-nitrophenoxide under the action of phospholipase and transacylase of LCAT, and the increase of absorbance at $400 \mathrm{~nm}\left(A_{400}\right)$ was determined to quantify the activity of LCAT. One unit of LCAT activity was defined as $1 \mu \mathrm{mol}$ of p-nitrophenoxide formed per hour, measuring the increase of $A_{400}$ expressed as $\mathrm{U} / \mathrm{mL}$ plasma (29). Plasma MPO activity was measured using the enzymatic colorimetric assay kit according to the instruction (Nanjing Jiancheng Bioengineering Institute).

\section{Plasma lipoprotein profiles analysis}

Plasma lipoprotein profiles were evaluated by FPLC using a Superose ${ }^{\mathrm{TM}} 6$ 10/300 GL column (17-5172-01, GE Healthcare Bio-Sciences, MA, USA) on an AKTA purifier (GE Healthcare Bio-Sciences) separated at a flow rate of $0.5 \mathrm{~mL} / \mathrm{min}$ in buffer $\left(10 \mathrm{mM} \mathrm{Na}_{2} \mathrm{HPO}_{4}, 0.15 \mathrm{M} \mathrm{NaCl}\right.$, $1 \mathrm{mM}$ EDTA) and fixed the fractionation volume to $500 \mu \mathrm{L}$ per tube. The TC content of lipoprotein fractions was determined by the method described above. The protein levels of PON1 and APOE in HDL fractions were detected by Western blot.

\section{Cell culture and incubated with PON1-containing plasma}

The RAW264.7 cells are monocyte/macrophagelike cell linage, which was obtained from Classic Specimen Culture and Storage Center at Wuhan University (Wuhan, China). The cells were cultured in Dulbecco's modified Eagle's medium (DMEM) supplemented with $10 \%(\mathrm{v} / \mathrm{v})$ FBS (Gibco, Thermo Fisher Scientific, MA, USA) and $1 \%$ penicillin/streptomycin (Biosharp, Hefei, China) at $37{ }^{\circ} \mathrm{C}$ in $5 \% \mathrm{CO}_{2}$ atmosphere. In order to detect the effect of PON1 on plasma HDL function in $S c a r b 1^{-/}$mice, HDLcontaining plasma was prepared by PEG-6000 (CAS 2532268-3, Sigma-Aldrich) precipitation method to remove apolipoprotein B (APOB)-containing LDL and very lowdensity lipoprotein (VLDL) (29). To detect the impact of HDL on cholesterol flux of macrophages, the RAW264.7 cells were loaded with $50 \mu \mathrm{g} / \mathrm{mL}$ ox-LDL for $12 \mathrm{~h}$, then incubated in fresh DMEM with 7\% APOB-depleted plasma for $24 \mathrm{~h}$. Lipid accumulation in cells was observed by Oil-Red $\mathrm{O}$ staining and cells integrated optical density (IOD) value measured by Image-Pro Plus 6.0 analysis. Additionally, C57BL/6 mice were intraperitoneally injected with $3 \mathrm{~mL} \mathrm{3 \%}$ thioglycolate, and peritoneal macrophages were harvested by peritoneal lavage after 3 days according to our previous method. For further assessment of the effect of plasma PON1 on related gene expression on macrophages, peritoneal macrophages were seeded in 6-well culture plates with $3 \times 10^{6}$ cells per well and incubated in DMEM with 7\% plasma separated from Lv-GFP mice or Lv-PON1 mice for $3 \mathrm{~h}$. The mRNA levels of macrophage genes were measured by quantitative real-time PCR (qRT-PCR).

\section{Histochemical and immunobistochemical analysis}

Hepatic PON1 expression was detected by immunohistochemistry. In brief, the liver sections were immobilized in 4\% paraformaldehyde for $20 \mathrm{~min}$, blocked in $5 \%$ BSA for $30 \mathrm{~min}$, and then incubated sequentially with the anti-PON1 antibody (1:500, ab24261, Abcam Inc., Cambridge, UK) and secondary antibody (HRP labeled). Finally, the sections were developed by DAB and the nuclei were counterstained with hematoxylin staining solution. The section image is visible under a microscope. The nucleus is stained blue by hematoxylin, and the positive $\mathrm{DAB}$ is brownish yellow.

Reactive oxygen species (ROS) levels in the liver were measured using the fluorescent dye DHE (D7008, SigmaAldrich) as described previously (29). Briefly, the liver cryosections were incubated with fluorescent dye DHE for $30 \mathrm{~min}$ and the nuclei were labeled after treatment with 4',6-Diamidino-2-phenylindole dihydrochloride (DAPI) (D9542, Sigma-Aldrich) for $10 \mathrm{~min}$. Images with red, green, and blue fluorescence were collected under a fluorescence microscope, respectively. ROS positive cells labeled by fluorescein were red, and the nuclei were blue by labeling with DAPI. The mean fluorescence intensity of DHE in the detected area was calculated using ImagePro Plus 6.0 and used to represent the ROS levels in liver sections.

In order to analyze the atherosclerotic lesions in the aorta, Oil-Red O staining was used to examine the $8 \mu \mathrm{m}$ serial cross-sections of the aortic root and en face of the aorta, and Image-Pro Plus 6.0 software was used to quantify the lesions (29). Besides, the lipid deposition of macrophages and cryosections of the liver were also detected by Oil-Red $\mathrm{O}$ staining. In addition, some paraffin sections of the liver were performed for hematoxylin and eosin (H\&E) staining and the morphology of liver tissue was observed under a microscope. 
Table 1 Primer sequences used for quantitative real-time polymerase chain reaction (qRT-PCR)

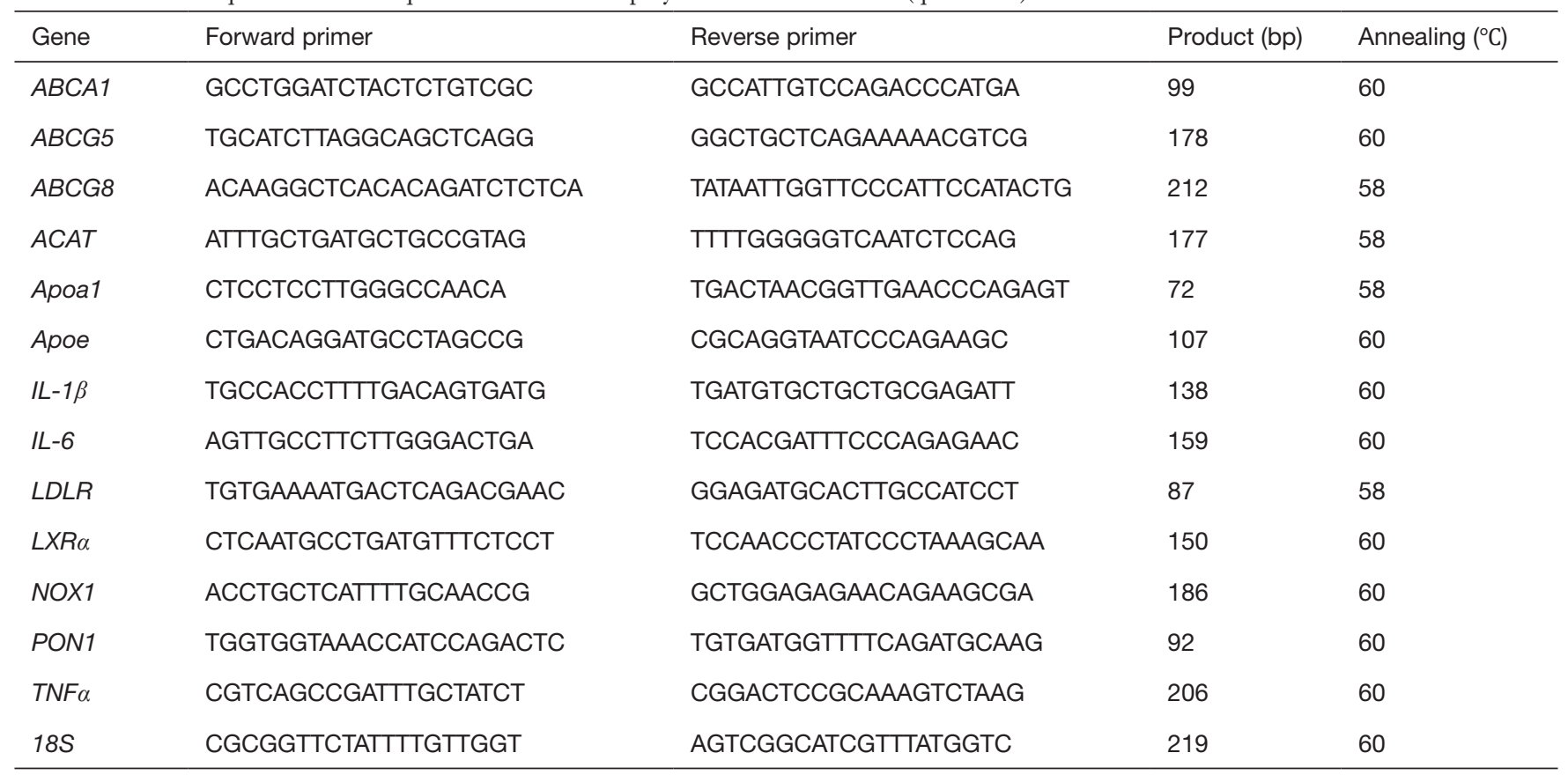

ABCA1, ATP-binding cassette A1; ABCG5/8, ATP-binding cassette G5/8; ACAT, acyl-coenzyme A cholesterol acyltransferase; Apoa1, apolipoprotein A1; Apoe, apolipoprotein E; bp, base pair; IL-1 $\beta$, interleukin-1 beta; IL-6, interleukin-6; NOX1, NADPH oxidase 1; LDLR, LDL receptor; LXR $\alpha$, liver X receptor alpha; NOX1, NADPH oxidase 1; PON1, paraoxonase1; TNF $\alpha$, tumor necrosis factor alpha.

\section{Lipids content analysis in liver}

About $50 \mathrm{mg}$ of liver tissues were homogenized and extracted with isopropanol overnight in tubes. After the isopropanol was completely evaporated with $\mathrm{N}_{2}$, the lipids were redissolved in a solution with the same volume. The amounts of TC, FC and TG were measured by the same method as the determination of blood lipids. The lipid content was normalized to the liver wet weight.

\section{Quantitative real-time PCR analysis}

Total RNA was extracted from cultured cells and liver using TRIzol Reagent (Invitrogen, CA, USA) according to the manufacturer's instructions. $1 \mu \mathrm{g}$ total RNA was reverse transcribed to cDNA with ReverTra Ace ${ }^{\circledR}$ qPCR RT Master Mix kit (TOYOBO, Osaka, Japan). The qRTPCR was performed using $\mathrm{SYBR}^{\circledR}$ Green Real-time PCR Master Mix (TOYOBO) on a CFX96 Touch $^{\text {TM }}$ RealTime PCR Detection System (Bio-Rad, CA, USA). The expression level of each gene was normalized to the level of $18 S$. The PCR primers for the amplification of mouse genes, including Apoa1, Apoe, PON1, Scarb1, LDL receptor
(LDLR), liver X receptor alpha (LXR $\alpha)$, ATP-binding cassette transporter A1 (ABCA1), ATP-binding cassette transporters G5/ G8 (ABCG5/G8), acyl-coenzyme A: cholesterol acyltransferase (ACAT), NADPH oxidase 1 (NOX1), interleukin-1 beta (LL-1ß), interleukin-6 (IL-6) and tumor necrosis factor alpha (TNFa) are shown in Table 1. The relative mRNA expression levels of genes were calculated using the $2^{\Delta \Delta \mathrm{Ct}}$ method.

\section{Western blot analysis}

The same volume of plasma or a certain amount of liver protein was separated by SDS-PAGE and transferred onto nitrocellulose (NC) membrane. The protein content of liver samples was determined using BCA protein assay kit (Thermo Fisher Scientific). Target proteins were specifically captured by primary antibodies and specific secondary horseradish peroxidase (HRP)-conjugated antibodies (Boster, Wuhan, China). The signals were detected using an enhanced chemiluminescence (ECL) kit (Advansta, CA, USA). The detailed information about the primary antibodies was listed in Table S1. Hepatic proteins levels were normalized using GAPDH as reference. 


\section{Statistical analysis}

Data are expressed as the mean \pm standard error of the mean (SEM). The number of experiment repetitions (n) was given in figure legends. GraphPad Prism software 5.0 (GraphPad Software, Inc., USA) was used for statistical analysis. Data between the two groups were compared by unpaired Student's $t$-test. A value of $\mathrm{P}<0.05$ was considered statistically significant.

\section{Results}

\section{Overexpression of PON1 plays a protective role in Scarb1 $1^{-/}$mice}

Lentiviruses Lv-PON1 or Lv-GFP were intravenously injected into $\mathrm{Scarb1}^{-1-}$ mice and maintained on a Western diet for 13 weeks. PON1 levels in liver tissues were firstly detected by immunohistochemical staining of cryosections, the result showed that hepatic expression of PON1 in Lv-PON1 mice was higher than that in Lv-GFP mice (Figure 1A). Meanwhile, Western blot results showed that the expression level of hepatic PON1 protein in Lv-PON1 mice was significantly increased by 1.1 folds versus $L v-G F P$ mice (Figure $1 B$ ). Since PON1 is a kind of secretory protein, Western blot analysis indicated that plasma PON1 level in Lv-PON1 mice was obviously increased by 2.6 times, compared to Lv-GFP mice (Figure $1 C$ ). It has been reported that abnormal HDL-C levels in $S_{c a r b 1}{ }^{-1-}$ mice shorten the lifespan of erythrocytes, leading to anemia and splenomegaly (30). In this study, we found that the spleen weight in Lv-PON1 mice was obviously lower than that of Lv-GFP mice. Plasma ALT activity was also improved in mice with high expression of PON1 in the liver, indirectly indicating that high expression of the antioxidant enzyme PON1 is conducive to maintaining the integrity of liver cells. There was no significant difference in body weight between the two groups of mice. Taking together, the results suggested that the overexpression of PON1 in the $S c a r b 1^{-1-}$ mice has no apparent toxicity but plays a protective role in vivo (Figure 1D). Furthermore, the DHE staining results of liver cryosections showed that the ROS level in the liver of Lv-PON1 mice was significantly lower than that of Lv-GFP mice by $18 \%$ (Figure $1 E$ ), which directly indicated that high-level PON1 reduced the oxidation state of the liver.

\section{PON1 overexpression decreased plasma FC level and improved plasma HDL-related functions}

Scarb1 $1^{-1-}$ mice had abnormally high cholesterol levels compared to wild-type mice. In order to investigate the impact of PON1 overexpression on plasma lipids, lipids levels in fasting plasma during 13 weeks of dietary induction were measured at the specified interval time-point. Although the plasma TC and TG levels of Lv-GFP mice and Lv-PON1 mice were not altered, the plasma FC level of Lv-PON1 mice was significantly lower than that of LvGFP mice (Figure 2A). The lipoprotein cholesterol profile separated by FPLC indicated that HDL-C in Lv-PON1 mice tended to be distributed in small HDL particles, while VLDL-C and LDL-C peaks were reduced, compared with those in the Lv-GFP group (Figure 2B). Furthermore, the relative distribution of PON1 and APOE in different HDL particles were measured by Western blot. The results showed that compared with the control group, the elevated APOE in Lv-PON1 mice was mainly distributed in large HDL particles, while the increased PON1 was mainly distributed in the small and medium HDL particles (Figure 2C). In addition, we explored the effects of PON1binding HDL on cultured macrophages. Macrophages were incubated with APOB-precipitation plasma isolated from two groups of Scarb1 $1^{-/}$mice, respectively. Oil-Red O staining of macrophages showed that treatment by plasma from the Lv-PON1 group reduced lipid accumulation in ox-LDL-stimulated cells, compared to the control treatment (Figure 2D). Meanwhile, the mRNA levels of related genes in peritoneal macrophages were measured. The data demonstrated that versus the control group, mRNA levels of cholesterol transporters Scarb1 and inflammatory cytokines $I L-6, T N F \alpha$ and NOX1 in macrophages were significantly decreased, while the mRNA level of $A B C A 1$ and anti-inflammatory cytokines $I L-4$ and $I L-10$ were increased after incubated for $3 \mathrm{~h}$ with the plasma from Lv-PON1 mice (Figure 2E). The above data indicated that PON1 highly expressed in the liver, could be secreted into plasma and bound to HDL, not only improved the esterification level of cholesterol and the structure of HDL particle in the circulation of Scarb $^{-1-}$ mice, but also increased the capacity of HDL to mediate cellular cholesterol efflux, as well as antioxidant and anti-inflammatory functions. 
A

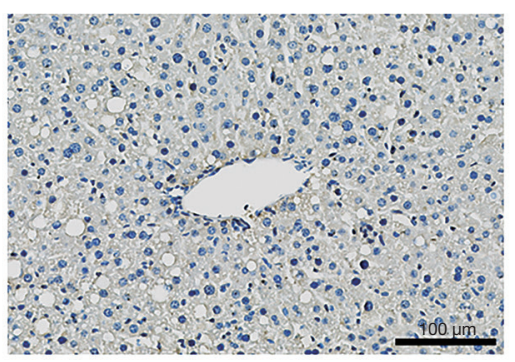

Lv-GFP

B
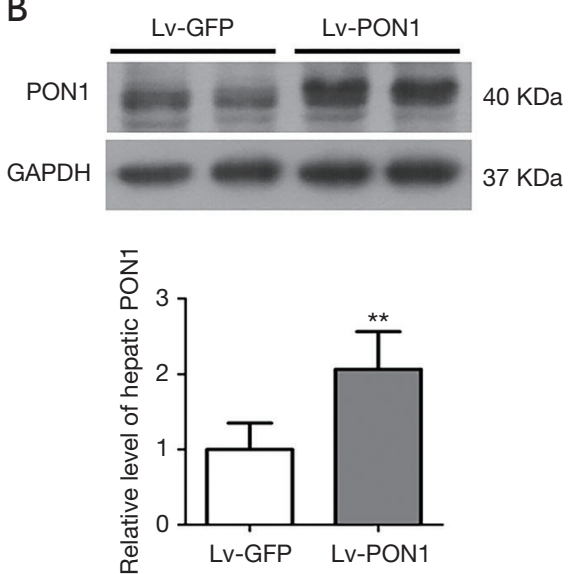

$\mathrm{E}$
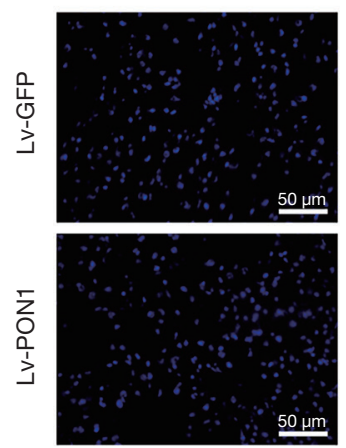

GFP
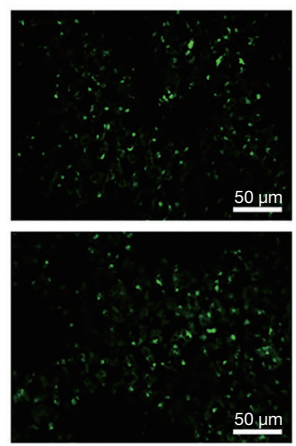

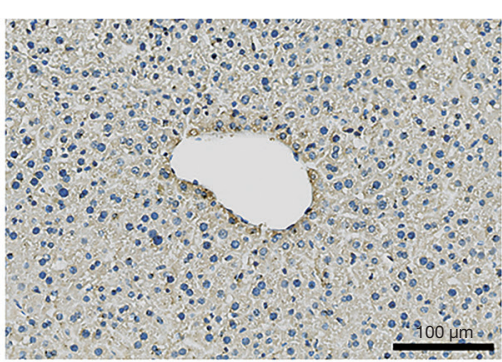

Lv-PON1

C

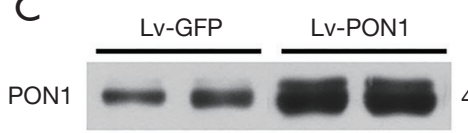
$40 \mathrm{KDa}$

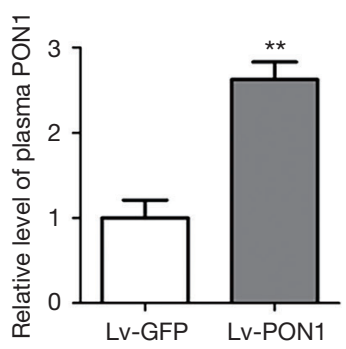

ROS
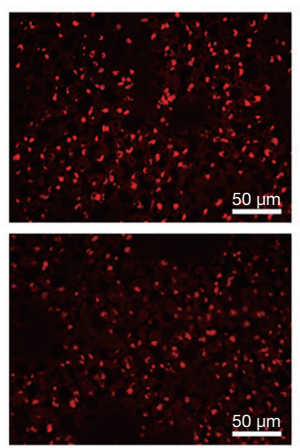

D
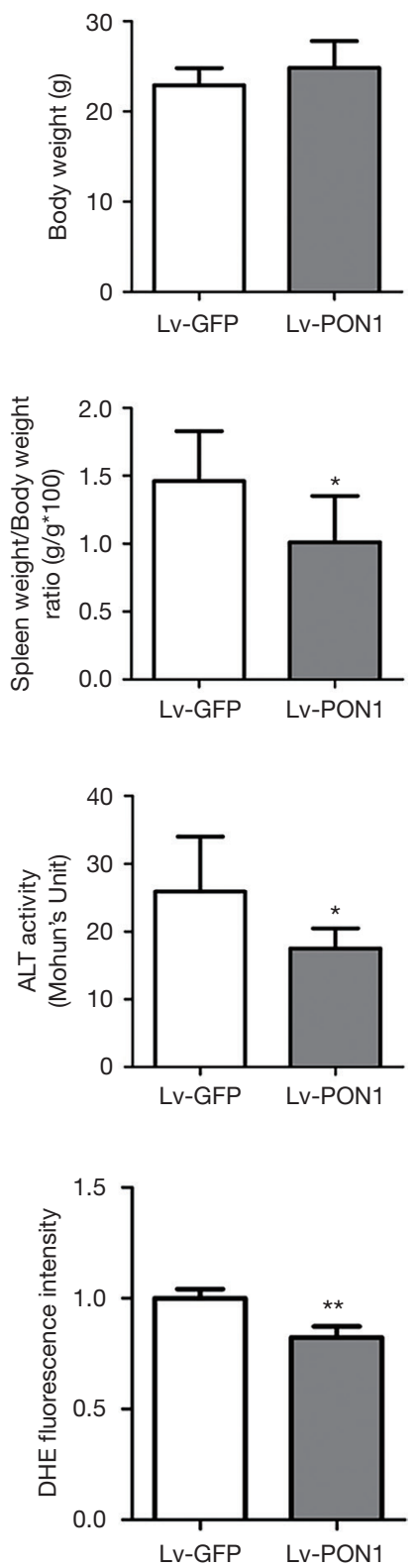

Figure 1 PON1 is overexpressed in the liver and secreted into the circulation in Scarb1 $1^{-/}$mice. (A) Expression of PON1 protein in the liver sections was determined by immunohistochemistry. Scale bars: $100 \mu \mathrm{m}$. (B) PON1 levels in the liver were normalized to GAPDH determined by Western blot analysis. $\mathrm{n}=4-5$ per group. (C) PON1 levels in the plasma were determined by Western blot analysis. n=9-10 per group. (D) Body weight, spleen weight/body weight ratio and plasma ALT activity were detected. $\mathrm{n}=10$ per group. (E) Representative pictures of ROS changes in mice liver were visualized by dihydroethidium (DHE) staining. Nuclei (blue), GFP (green) and DHE (red) were shown. The ROS levels were calculated using Image-Pro Plus 6.0 and represented by the mean DHE fluorescence intensity in the detected area. Scale bars: $50 \mu \mathrm{m}$. The measurement data were presented as mean \pm standard error of the mean. Data between the two groups were compared by unpaired Student's $t$-test. ${ }^{*} \mathrm{P}<0.05,{ }^{* *} \mathrm{P}<0.01$, versus Lv-GFP group. 
A
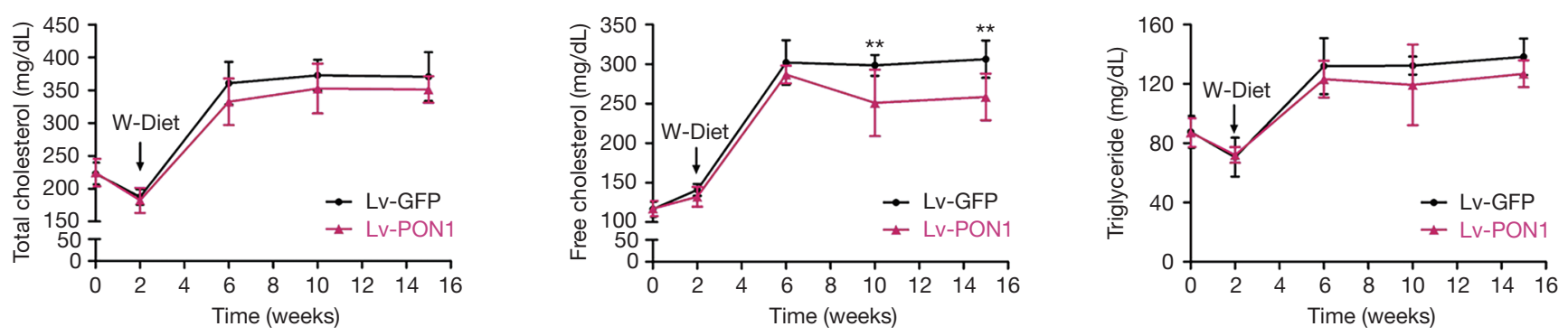

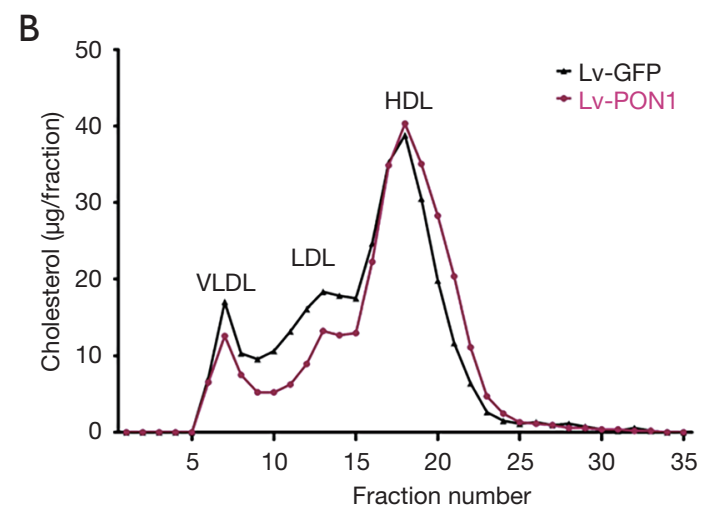

C

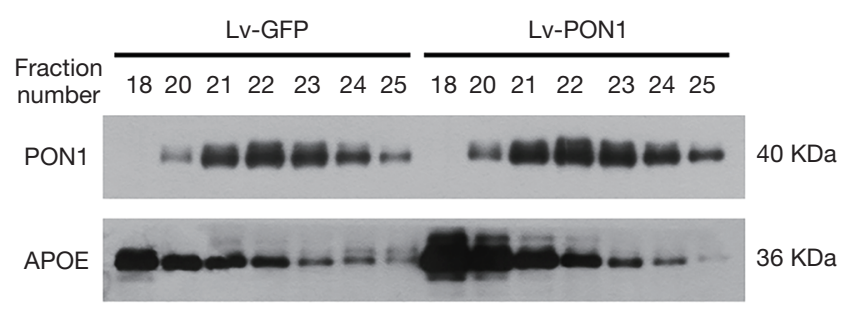

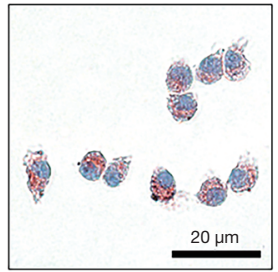

Lv-GFP

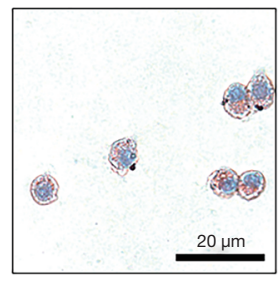

Lv-PON1

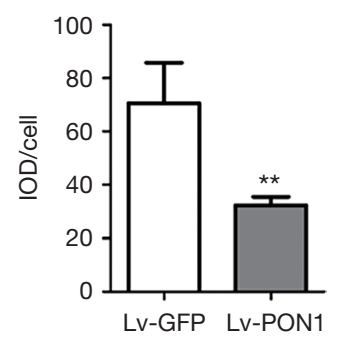

$\mathrm{E}$

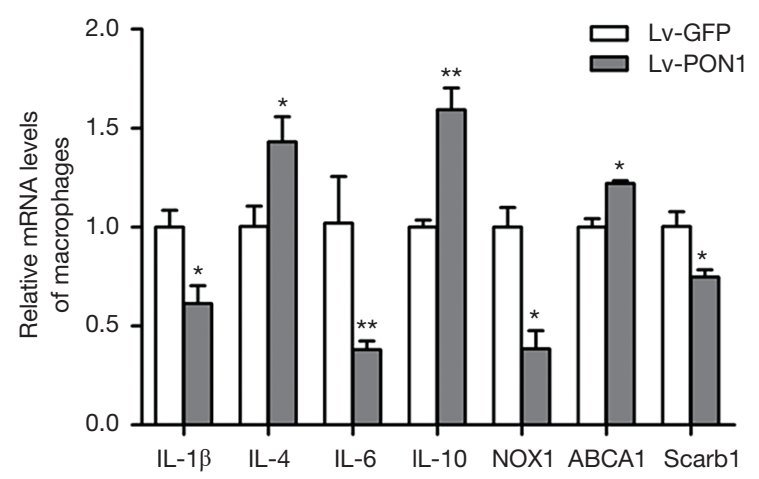

Figure 2 PON1 overexpression decreased plasma FC level and improved plasma HDL-related functions. (A) TC, FC, and TG levels of fasting plasma collected at the indicated time were determined enzymatically as described in Methods. $n=9-10$ per group. (B) Lipoprotein profiles in pooled plasma were size fractionated by FPLC using a Superose ${ }^{\mathrm{TM}} 6$ 10/300 GL column, and cholesterol contents within lipoprotein fractions (VLDL, LDL, and HDL) were detected. (C) HDL functional protein levels at the HDL peak collected by FPLC were measured by Western blot analysis. (D) Macrophages were treated with APOB-depleted plasma, which was separated from Scarb $1^{-/-}$mice injected lentivirus. The intracellular lipid contents were detected using Oil Red O-stained and cells integrated optical density (IOD) value measured by Image-Pro Plus 6.0 analysis. Scale bars: $20 \mu \mathrm{m}$. Data were from three independent experiments. (E) Peritoneal macrophages were treated with APOB-depleted plasma, which was separated from Scarb1 $1^{-/}$mice injected lentivirus. The relative mRNA levels of genes in macrophages were determined by qRT-PCR. mRNA levels of target genes were normalized to that of $18 \mathrm{~S}$, which was selected as an internal standard. $\mathrm{n}=3$ per group. The measurement data were presented as mean \pm standard error of the mean. Data between the two groups were compared by unpaired Student's $t$-test. ${ }^{*} \mathrm{P}<0.05$, ${ }^{*} \mathrm{P}<0.01$, versus $\mathrm{Lv}$-GFP group. qRT-PCR, quantitative real-time polymerase chain reaction. 
A

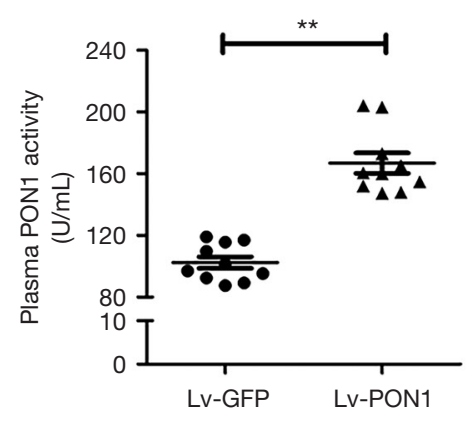

$\mathrm{D}$

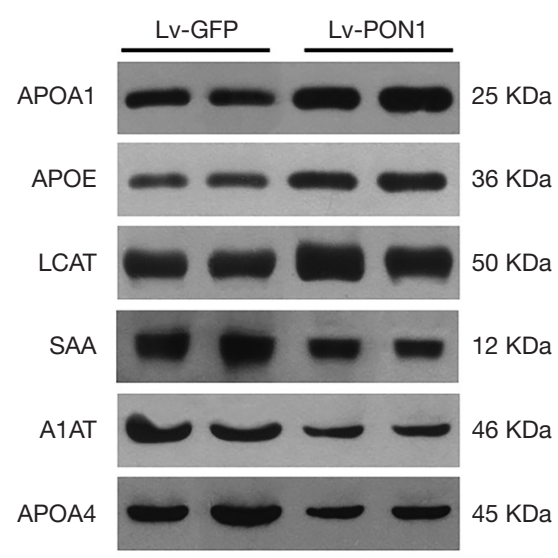

B

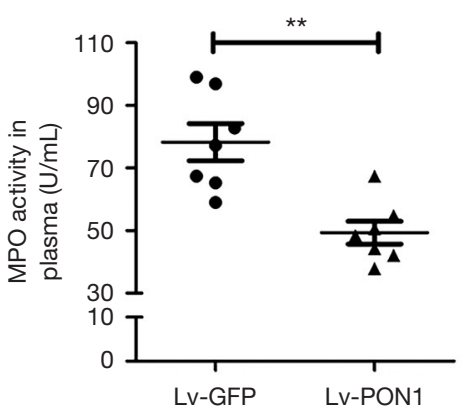

C

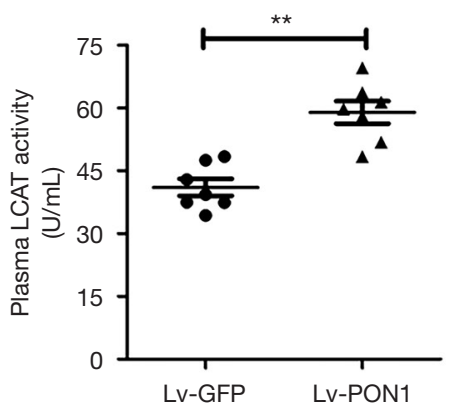

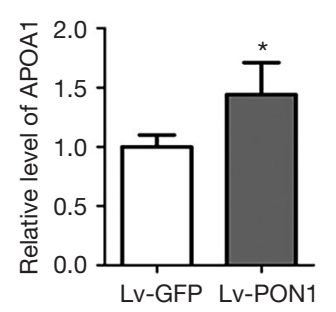
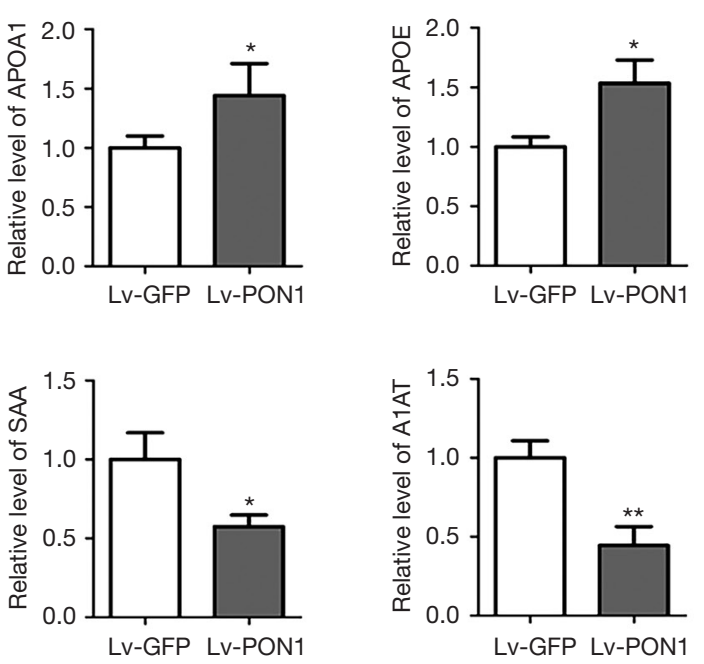
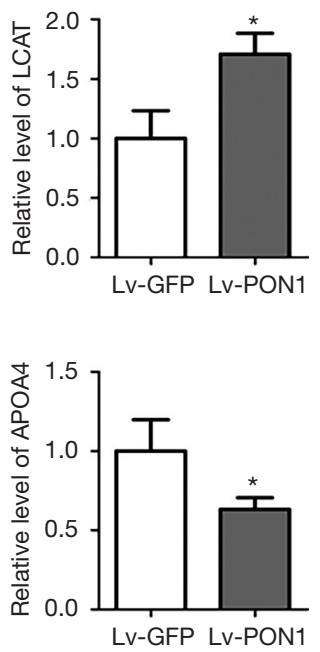

Figure 3 PON1 induces an atheroprotective HDL protein-profile. (A) Plasma PON1 activity was determined. n=10 per group. (B) and (C) Plasma MPO and LCAT activities were determined by enzymatic colorimetric assay. $n=7$ per group. (D) Plasma HDL-associated protein levels of APOA1, APOE, LCAT, SAA, A1AT and APOA4 were detected by Western blot. $\mathrm{n}=3-4$ per group. The measurement data were presented as mean \pm standard error of the mean. Data between the two groups were compared by unpaired Student's $t$-test. ${ }^{*} \mathrm{P}<0.05$, **P $<0.01$, versus Lv-GFP group.

\section{High-level PON1 improves HDL-associated proteins}

To further evaluate the effect of PON1 on protein components of dysfunctional HDL in $\mathrm{Scarb1}^{-/-}$mice, we detected some enzyme activities and protein levels related to HDL function. Firstly, consistent with the increase in plasma PON1 level, plasma PON1 activity in the Lv-PON1 group was about 1.6 times higher than that in the Lv-GFP control group $(102.5 \pm 12.0$ vs. $166.8 \pm 20.9 \mathrm{U} / \mathrm{mL}, \mathrm{P}<0.01)$ (Figure $3 A$ ). MPO is a highly oxidative enzyme secreted by activated neutrophils, monocytes and macrophages. In contrast to the antioxidant function of PON1, MPO plays a role in the oxidative modification of circulating and subintimal lipoproteins. We found that compared with the control mice, the MPO activity of the Lv-PON1 group was markedly decreased $(85.5 \pm 8.3$ vs. $57.3 \pm 8.2 \mathrm{U} / \mathrm{mL}, \mathrm{P}<0.01)$ (Figure $3 B$ ). In addition, the plasma FC/TC ratio of Scarb1 ${ }^{-1-}$ mice was increased significantly, one of the reasons was that the activity of LCAT decreased, which inhibited the esterification of FC. Our data showed that compared to the control group, not only plasma LCAT protein level was increased by 1.7 times, but also plasma LCAT activity was significantly increased in Lv-PON1 mice $(41.1 \pm 5.4$ vs. 59.0 \pm 7.0 U/mL, $\mathrm{P}<0.01$ ) (Figure 3C,D). Furthermore, we measured the levels of several valuable proteins that in the previous study had been considered as biomarkers for dysfunctional HDL in Scarb1 $1^{-/}$mice. Western blot analysis 


\section{Page 10 of 17}

showed that compared with Lv-GFP mice, the levels of HDL functional proteins APOA1 and APOE in plasma were increased, while the levels of acute-phase proteins SAA, A1AT and APOA4 were significantly decreased in Lv-PON1 mice (Figure 3D). These results indicated that raising PON1 activity in circulation can increase plasma HDL-associated functional protein levels, promote HDL esterification, and improve the oxidation and inflammation status of HDL.

\section{PON1 overexpression attenuates atherosclerosis and bepatic steatosis}

To observe the impact of PON1 overexpression on atherosclerosis, Oil-Red O staining of the en face aorta and cross-sections of aortic roots were performed after 13 weeks of Western diet feeding. Since Scarb1 ${ }^{-1-}$ mice developed infiltrating atherosclerotic lesions, representative images of atherosclerotic lesions in cross-sections of aortic roots and en face aorta were shown in figure (Figure 4A,B). Quantitative analysis revealed that compared with $\mathrm{Lv}-$ GFP mice, the infiltrated lesion area of en face aorta in LvPON1 mice was reduced by nearly 34\% (Figure 4C), and the mean lesion area of aortic roots in $\mathrm{Lv}-\mathrm{PON} 1$ mice also showed a significant decrease $(0.093 \pm 0.017$ vs. $0.052 \pm 0.011$ $\mathrm{mm}^{2}, \mathrm{P}<0.01$ ) (Figure $4 D$ ). Moreover, overexpression of hepatic PON1 markedly reduced the lipid deposition in the liver, as revealed by $\mathrm{H} \& \mathrm{E}$ staining and Oil-Red $\mathrm{O}$ staining (Figure $4 E$ ) or by measurement of liver lipid contents. Interestingly, the data indicated that versus Lv-GFP mice, hepatic FC level in Lv-PON1 mice was significantly decreased, while the contents of TC and TG were not altered (Figure 4F). The above data suggest that hepatic PON1 overexpression may contribute to the reduction of atherosclerosis and liver steatosis.

\section{PON1 regulates the expression of hepatic genes related to HDL metabolism and inflammation}

In order to clarify the possible mechanism of hepatic PON1 expression to improve HDL metabolism, we further detected the expression levels of some important genes involved in the regulation of HDL biogenesis, hepatic cholesterol uptake, and cholesterol conversion and excretion by qRT-PCR and Western blot. We found from qRTPCR data that compared to the Lv-GFP mice, the mRNA levels of Apoa1, Apoe, LDLR, $L X R \alpha, A B C A 1, A B C G 5 / 8$, and $A C A T$ were remarkably increased in the liver of $\mathrm{Lv}-\mathrm{PON} 1$

\section{Zhao et al. PON1 on dysfunctional HDL in Scarb1 deficient mice}

mice (Figure 5A). Consistently, the data from Western blot showed that the protein levels of APOA1, APOE, LDLR, $\mathrm{LXR} \alpha, \mathrm{ABCA} 1, \mathrm{ABCG} 5 / 8$, and ACAT were also elevated in Lv-PON1 mice (Figure 5B).

Additionally, NF- $\kappa \mathrm{B}$ was identified as a ROS-inducible target, which up-regulated the expression of $I L-6$ and $T N F \alpha$. Therefore, the NF- $\kappa \mathrm{B}$ mediated inflammatory pathway in the liver was measured. Combined with the results of qRTPCR and Western blot (Figure $5 A, B$ ), the data indicated that PON1 overexpression distinctly down-regulated the mRNA levels of $I L-6$ and $T N F \alpha$ by suppressing the phosphorylation

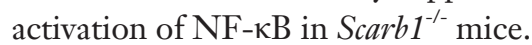

\section{Discussion}

There is increasing evidence that dysfunctional HDL is an important proatherogenic factor (5). In this study, Scarb $^{-/-}$mice characterized by dysfunctional HDL, were intravenously injected with lentiviruses to achieve PON1 overexpression in the liver and the increase of PON1 level and activity in the circulation. We found that hepatic PON1 overexpression significantly reduced the oxidative and inflammatory status in vivo, improved dysfunctional HDL composition and function, indirectly promoted HDL biogenesis and maturation, and accelerated cholesterol metabolism, thereby resulting in the reduction of hepatic steatosis and atherosclerotic lesions in Western diet-fed Scarb $1^{-/}$mice. These pleiotropic effects strongly suggest that PON1 is an endogenous regulator of HDL structure and function through its antioxidase activity, and may be a prospective therapeutic target for the treatment of dysfunctional HDL-related atherosclerotic cardiovascular disease.

PON1 is secreted from the liver into the blood and is considered to be one of the most important components of HDL antioxidant performance $(12,15)$. Different epidemiological studies have explored the relationship between PON1 functional activity and gene polymorphisms (e.g., rs662, rs854560) (31), providing direct evidence that PON1 activity influenced by genotypes is involved in the development of chronic inflammatory diseases, such as atherosclerotic cardiovascular disease, diabetes mellitus, and neurodegenerative diseases (32). However, there is no direct evidence to explain the specific effect and mechanism of PON1 on HDL metabolism. we previously identified a reduction in PON1 level and activity as a biomarker for dysfunctional HDL in Scarb1 $1^{-/}$mice (25), therefore, from the perspective of active repair, the present study 
A

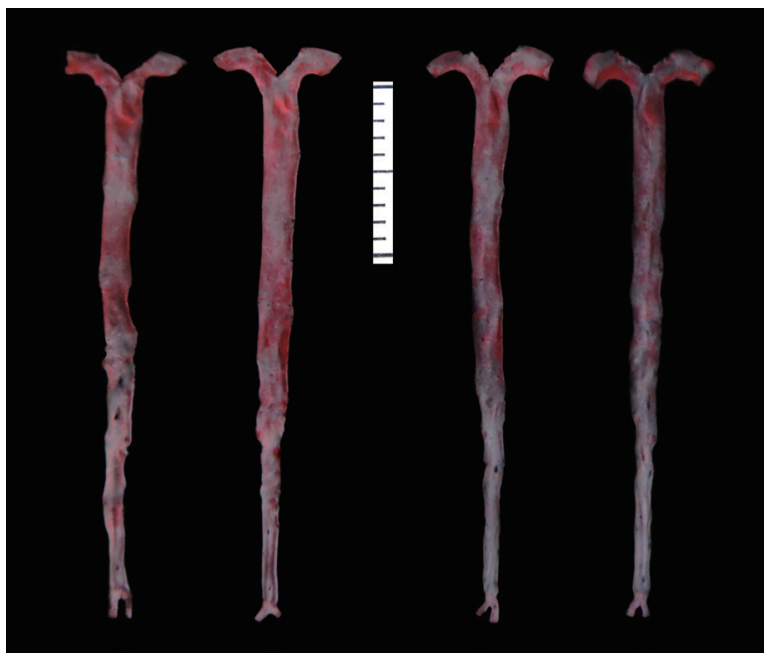

Lv-GFP

Lv-PON1

$E$

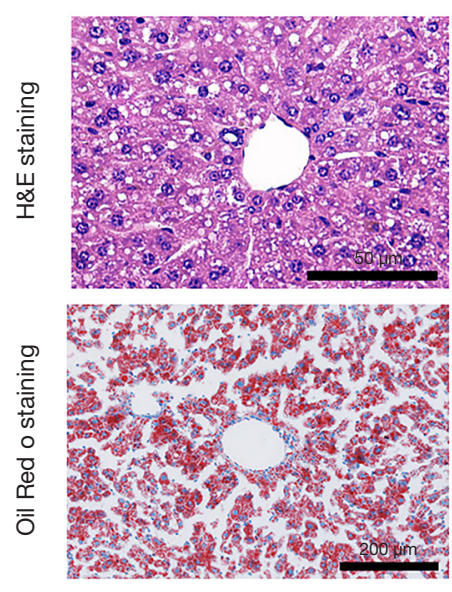

Lv-GFP

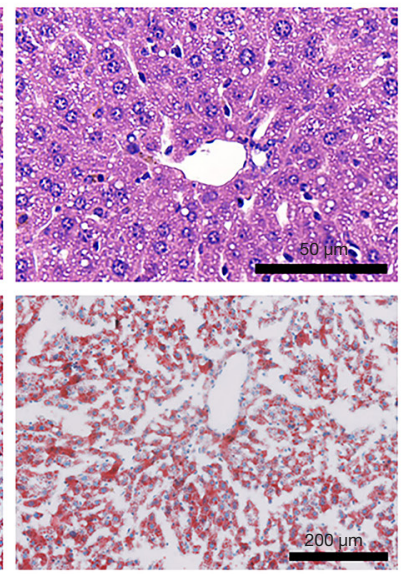

Lv-PON1
B

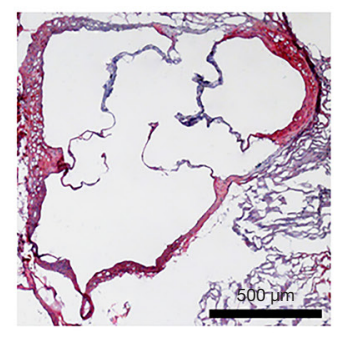

Lv-GFP

C

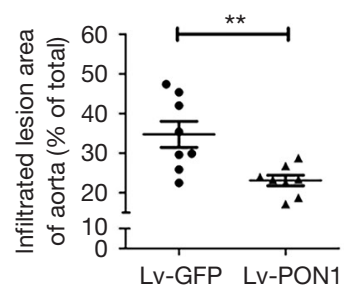

$\mathrm{F}$

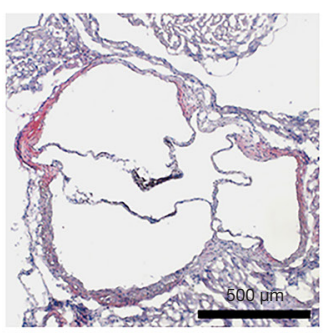

Lv-PON1

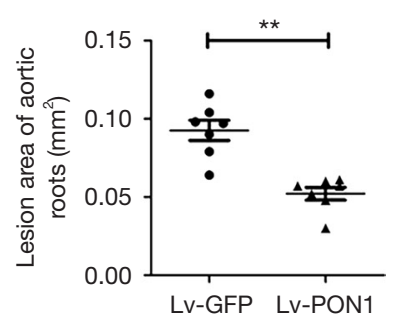

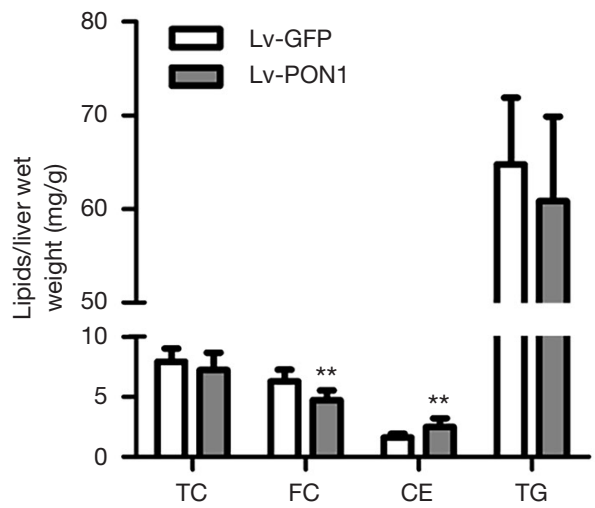

Figure 4 PON1 overexpression attenuates atherosclerosis and hepatic steatosis in $S c a r b 1^{-/-}$mice. (A) Representative images of atherosclerotic lesions by en face aortas Oil Red O-staining. (B) Atherosclerotic lesions were examined by Oil Red O-stained cross-sections of the aortic root (8 $\mu \mathrm{m}$ serial sections). Scale bars: $500 \mu \mathrm{m}$. (C) Relative quantification of lesion area in the Lv-GFP and Lv-PON1 mice, data are presented as the percentages of en face aortic area. $n=8$ per group. (D) The cross-sectional lesion areas of the aortic roots were quantified. The data are presented as the lesion areas $\left(\mathrm{mm}^{2}\right)$. $\mathrm{n}=7$ per group. (E) Hepatic lipid levels were analyzed by hematoxylin and eosin (H\&E) or Oil Red O staining. Scale bars: 50 and $100 \mu \mathrm{m}$, respectively. (F) The contents of TC, FC and TG were determined using isopropanol as described in Methods. $n=8-9$ per group. The measurement data were presented as mean \pm standard error of the mean. Data between the two groups were compared by unpaired Student's $t$-test. ${ }^{* *} \mathrm{P}<0.01$, versus Lv-GFP group.

originally observed that overexpression of PON1 in the liver positively improved the level and activity of PON1 in the liver and the circulation. Highly active PON1 significantly decreased ROS levels and NF-кB-mediated inflammatory factors IL- 6 and TNF- $\alpha$ expression levels in the liver. Not only that, high-level PON1 which binding with HDL in circulation could participate in the regulation of HDL functions, including decreasing macrophage lipid accumulation by facilitating ABCA1-mediated cholesterol efflux, and inhibiting oxidase activity and inflammatory 


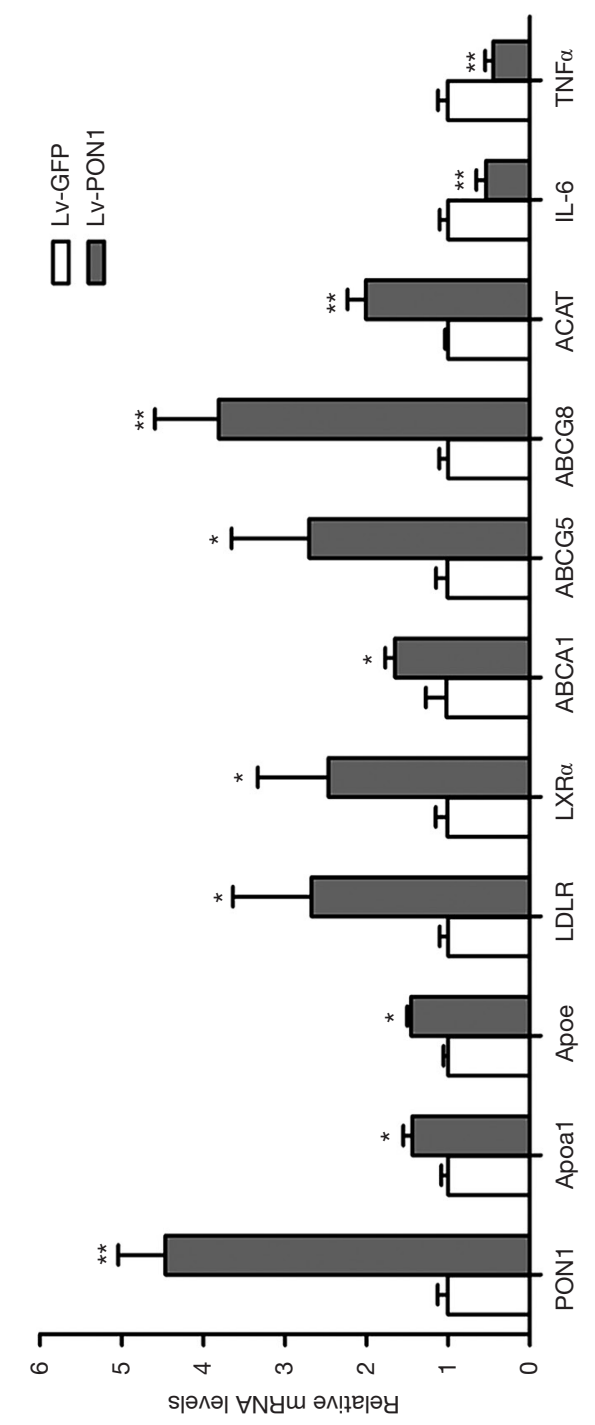

$\varangle$
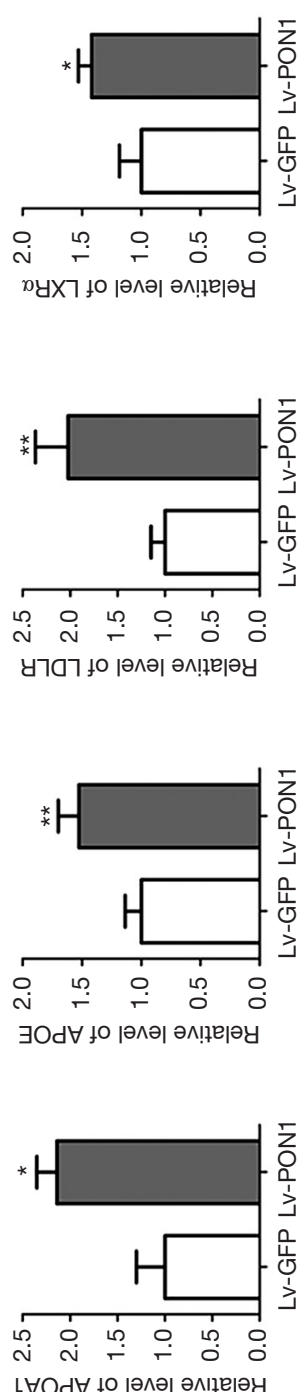

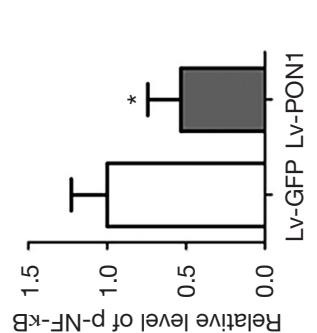

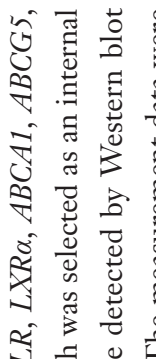

궁

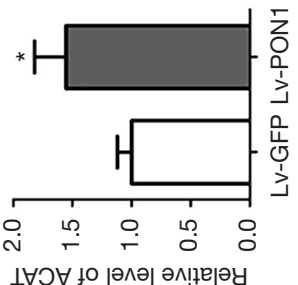

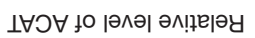

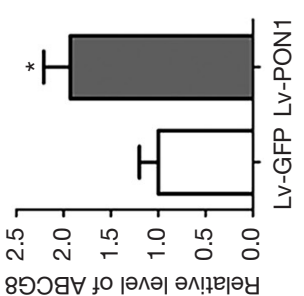

8९วЯ

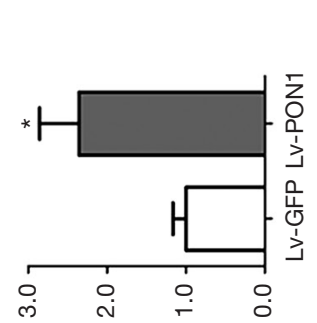

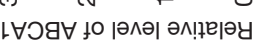

उั

空

¿

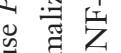

它古

$\sum$ च

य)

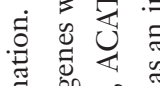

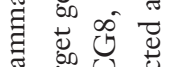

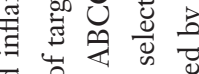

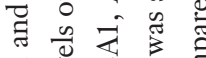

苟总它

《यद

उ $Z$

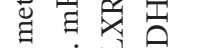

过完

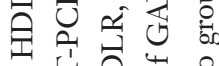

.

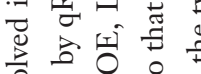

응 0
Ч

岩 官 50 空

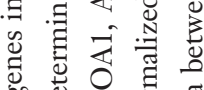

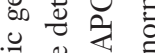

.

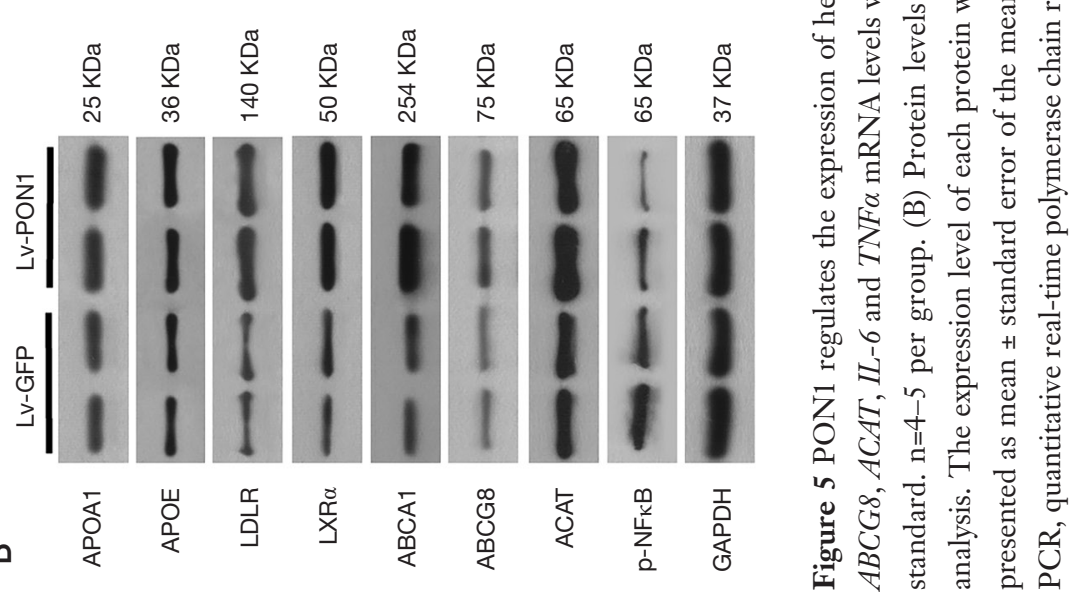


response of macrophages through reducing $I L-1 \beta, I L-6$ and NOX1 expression levels, and increasing $I L-4$ and $I L-10$ expression levels.

In addition, earlier in vitro studies have revealed that oxidative modification of HDL or APOA1 catalyzed by MPO can shift HDL into pro-inflammatory particles (33). Serum MPO/PON1 ratio was considered as a potential indicator of dysfunctional HDL and risk stratification in coronary artery disease. An interesting study proposed that PON1 can form a tertiary complex with HDL and MPO which can modulate the activity of MPO (34). Our previous study showed increased plasma MPO activity in Scarb1 $1^{-1-}$ mice (25). Consistent with the former findings, this study supported that the high level of PON1 was related to the decrease of MPO activity in plasma, which provided strong evidence that PON1 could improve APOA1 function and reduce the oxidative and inflammatory status of HDL in Scarb $1^{-/}$mice.

It has been recognized that circulating HDL particles are substantial heterogeneity in the composition of lipids and proteins due to their complex metabolism. However, experimental studies have also shown that the antiatherogenic properties of HDL were determined by protein components. Consistent with the improvement of HDL functions by PON1, our current studies specifically demonstrated that PON1 overexpression significantly altered the composition of HDL, represented with the increase of functional proteins such as APOA1, APOE and LCAT, while the reduction of acute-phase protein SAA, APOA4 and A1AT as pro-inflammatory biomarkers in $\mathrm{Scarb1}^{-/}$mice. This experimental evidence further supported that PON1 could repair dysfunctional HDL and play an important role in maintaining the structure and function of normal HDL.

APOA1, as the main apolipoprotein of HDL, plays a vital role in HDL-mediated RCT and also has anti-inflammatory and antioxidant functions. APOA1 reacts specifically with ABCA1 in the liver is to generate new HDL particles, while the effect on macrophages is to induce cholesterol efflux from the cells. Meanwhile, the stable and high affinity between PON1 and HDL requires interaction with APOA1 (35). However, studies also confirmed that APOA1 suffered from functional impairment due to oxidative modification under various inducements and accumulated in atherosclerotic plaques (36). A recent study reported a significant increase of APOA1 oxidation in human SCARB1 heterozygote carriers and patients with coronary artery disease (37). This suggests that increasing the level and reducing the oxidation of APOA1 might enhance the antiatherogenic function of APOA1. Our results showed that high-level PON1 not only increased plasma APOA1 level, but also reduced the degree of APOA1 oxidation due to the antioxidant effects of PON1 and lower MPO activity, thus enhancing the protective effect of APOA1 on the blood vessel wall. Although we did not directly detect the oxidation state of APOA1, the anti-atherogenesis effect of PON1 by acting on APOA1 can be indirectly confirmed by the experiment that APOA1/HDL-containing plasma increased the cholesterol efflux of macrophages.

APOE is another major apolipoprotein in HDL particles and an important ligand of LDLR, which mediates the clearance of APOE-containing lipoproteins from the circulation through the hepatic LDLR pathway (38). It also promotes cholesterol efflux from macrophages and exerts a protective effect on oxidative processes and inflammation (39). In the Scarb1 ${ }^{-1-}$ mouse model we used, although the HDL-mediated RCT pathway was hindered due to SR-BI deficiency, another hepatic cholesterol uptake pathway was enhanced, manifested by significantly increased expression levels of APOE and LDLR in the liver. That is to say, high-level PON1 promoted hepatic uptake of APOE-containing lipoproteins (VLDL and HDL) by the APOE-LDLR-mediated pathway, thus explaining the tendency of abnormally large HDL particles to become smaller and the decrease in plasma levels of lipoprotein remnants. These effects are beneficial to reduce the development of atherosclerosis in Scarb $1^{-/}$mice.

Plasma LCAT activated by APOA1 is a key enzyme catalyzing the esterification of $\mathrm{FC}$ into $\mathrm{CE}$, which plays a crucial role in HDL maturation (40). It has also been reported that LCAT has the similar functional activity to PON1 and hydrolyze oxidized phospholipids (41). The previous findings revealed that subjecting human plasma to oxidative stress reduced the activity of LCAT (42). Meanwhile, LCAT activity in Scarb1 $1^{-1}$ mice was reduced, resulting in the decrease of endogenous esterification ratio (43). Our current work showed that increasing plasma PON1 antioxidant activity promoted FC esterification by elevating the level and activity of plasma LCAT, which leads to the decrease of the abnormally high level of FC in $\mathrm{Scarb}^{-/-}$mice, thereby, providing an indirect mechanism for PON1 to repair dysfunctional HDL through LCATcatalyzed HDL maturation and reduction of oxidized lipids.

Liver is the central metabolic organ and plays a pivotal role in HDL metabolism and cholesterol metabolism, including HDL biogenesis, HDL-C selective uptake and 


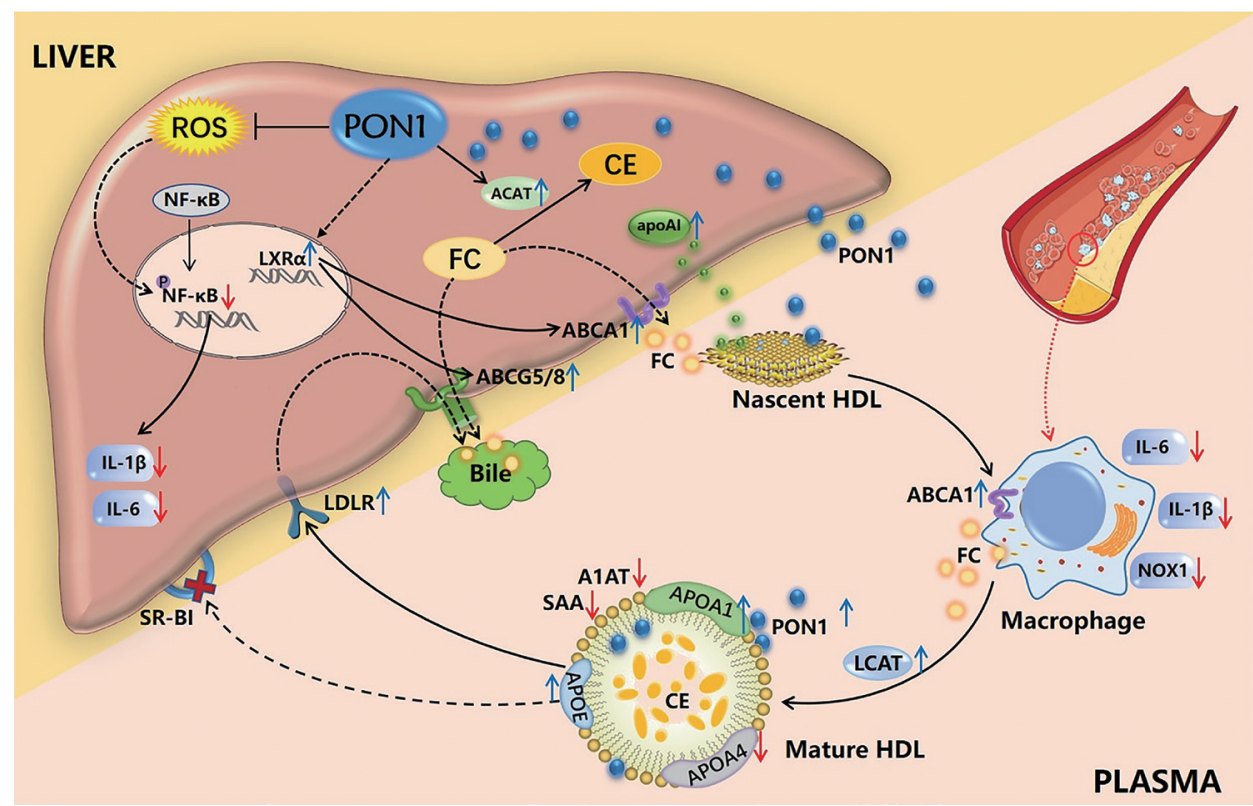

Figure 6 The pleiotropic effect of high-level PON1 on liver and circulation in Scarb1 $1^{-/}$mice. Hepatic PON1 overexpression and secretion into the circulation reduced the oxidation and inflammation status of dysfunctional HDL, improved the composition of HDL, promoted HDL biogenesis and maturation, and enhanced hepatic cholesterol uptake and excretion into the bile, which contributed to alleviating hepatic steatosis and atherosclerotic lesions.

conversion, and excretion from bile (44). The regulation of PON1 in the circulation is inseparable from its influence on the liver. Therefore, we explore the changes in the expression of genes related to cholesterol metabolism from the perspective of redox regulation. Firstly, we were delighted to observe that PON1 not only reduced the inflammatory status of the liver, but also stimulated the synthesis of HDL functional components APOA1 and APOE to a certain extent, which was consistent with the increase in plasma levels of APOA1 and APOE. Beyond that, it is documented that cholesterol efflux pathways mediated by ATP-binding cassette transporters and HDL suppress atherosclerosis (45). The role of ABCA1 in the liver is to export cellular cholesterol to lipid-free APOA1 for the biogenesis of nascent HDL (46), while the heterodimer of ABCG5 and ABCG8 is responsible for the excretion of cholesterol into bile (47). It's worth noting that LXRa is a metabolic nuclear receptor that transcriptionally upregulates ABCA1 and ABCG5/G8 (48). As previously reported, that reduced ROS could trigger $\mathrm{LXR} \alpha$ activation. Our current studies indicated that PON1 promoted hepatic cholesterol movement by LXRa-mediated upregulation of ABCA1 and ABCG5/8, which may be due to the fact that PON1 improved oxidation and inflammation in the liver, leading to the increase of LXR $\alpha$ level. Additionally, studies also provided information that LXRa could serve as an important up-regulator of APOE, LDLR and SR-BI, as well as a down-regulator of NF- $\mathrm{kB}$-mediated inflammatory pathway $(49,50)$, which are consistent with our findings, except for SR-BI deficiency. Besides, we also observed that the level of hepatic ACAT was elevated, which catalyzed cytoplasm FC esterification (51). It is beneficial to reduce the lipotoxicity of FC to hepatocytes in $S c a r b 1^{-1-}$ mice. Taken together, these results demonstrated that hepatic PON1 overexpression decreased the FC level in plasma and liver, and alleviated the deposition in the liver of Scarb1 ${ }^{-1-}$ mice.

In conclusion, our present study used a mouse model of dysfunctional HDL to prove that the overexpression and secretion of PON1 could directly and indirectly reduce the oxidative and inflammatory properties of HDL, improve HDL protein components, and regulate cholesterol metabolism through pleiotropic effects, including the promotion of HDL biogenesis and maturation, cholesterol uptake by the liver and excretion into the bile, thereby reducing atherosclerotic lesions and hepatic steatosis in $\mathrm{Scarb1}^{-1-}$ mice fed on a Western diet (Figure 6). Although the SR-BI pathway is blocked in model mice, the results 
achieved by improving the level and activity of PON1 once again emphasize the importance of HDL antioxidant function in reducing atherosclerosis. These findings provide valid evidence that targeting PON1 represents a promising HDL-based therapeutic strategy for cardiovascular disease.

\section{Acknowledgments}

Funding: This work was supported by the National Natural Science Foundation of China (No.81670423, No.82070471 and No. 81270364 to HY); the Wuhan Science and Technology Bureau (Hubei Province) (No. 2019020701011489 to DFW); and the Medical Science Advancement Program (Basic Medical Science) of Wuhan University (TFJC2018001 to HY). The manuscript revision was in part by grants from Translational Medicine Joint Fund of Zhongnan Hospital of Wuhan University (ZNLH201907 to DFW).

\section{Footnote}

Reporting Checklist: The authors have completed the ARRIVE reporting checklist. Available at https://dx.doi. org/10.21037/atm-21-682

Data Sharing Statement: Available at https://dx.doi. org/10.21037/atm-21-682

Conflicts of Interest: All authors have completed the ICMJE uniform disclosure form (available at https://dx.doi. org/10.21037/atm-21-682). The authors have no conflicts of interest to declare.

Ethical Statement: The authors are accountable for all aspects of the work in ensuring that questions related to the accuracy or integrity of any part of the work are appropriately investigated and resolved. Animal experiments were performed under a project license (No. 2018027, 19 March 2018) granted by the Medical Animal Care and Welfare Committee for Animal Experiments of Wuhan University. All experiments were performed according to the guidelines for the care and use of laboratory animals of the Chinese Animal Welfare Committee.

Open Access Statement: This is an Open Access article distributed in accordance with the Creative Commons Attribution-NonCommercial-NoDerivs 4.0 International License (CC BY-NC-ND 4.0), which permits the non- commercial replication and distribution of the article with the strict proviso that no changes or edits are made and the original work is properly cited (including links to both the formal publication through the relevant DOI and the license). See: https://creativecommons.org/licenses/by-nc-nd/4.0/.

\section{References}

1. Libby P, Ridker PM, Hansson GK. Progress and challenges in translating the biology of atherosclerosis. Nature 2011;473:317-25.

2. März W, Kleber ME, Scharnagl H, et al. HDL cholesterol: reappraisal of its clinical relevance. Clin Res Cardiol 2017;106:663-75.

3. Van Lenten BJ, Hama SY, de Beer FC, et al. Antiinflammatory HDL becomes pro-inflammatory during the acute phase response. Loss of protective effect of HDL against LDL oxidation in aortic wall cell cocultures. J Clin Invest 1995;96:2758-67.

4. Kontush A, Chapman MJ. Functionally defective high-density lipoprotein: a new therapeutic target at the crossroads of dyslipidemia, inflammation, and atherosclerosis. Pharmacol Rev 2006;58:342-74.

5. Rosenson RS, Brewer HB, Jr., Ansell BJ, et al. Dysfunctional HDL and atherosclerotic cardiovascular disease. Nat Rev Cardiol 2016;13:48-60.

6. Stoekenbroek RM, Stroes ES, Hovingh GK. ApoA-I mimetics. Handb Exp Pharmacol 2015;224:631-48.

7. Valanti EK, Chroni A, Sanoudou D. The future of apolipoprotein $\mathrm{E}$ mimetic peptides in the prevention of cardiovascular disease. Curr Opin Lipidol 2019;30:326-41.

8. Gan KN, Smolen A, Eckerson HW, et al. Purification of human serum paraoxonase/arylesterase. Evidence for one esterase catalyzing both activities. Drug Metab Dispos 1991;19:100-6.

9. Costa LG, Cole TB, Jarvik GP, et al. Functional genomic of the paraoxonase (PON1) polymorphisms: effects on pesticide sensitivity, cardiovascular disease, and drug metabolism. Annu Rev Med 2003;54:371-92.

10. Watson AD, Berliner JA, Hama SY, et al. Protective effect of high density lipoprotein associated paraoxonase. Inhibition of the biological activity of minimally oxidized low density lipoprotein. J Clin Invest 1995;96:2882-91.

11. Aviram M, Rosenblat M, Bisgaier CL, et al. Paraoxonase inhibits high-density lipoprotein oxidation and preserves its functions. A possible peroxidative role for paraoxonase. J Clin Invest 1998;101:1581-90.

12. Rosenblat M, Gaidukov L, Khersonsky O, et al. The 


\section{Page 16 of 17}

catalytic histidine dyad of high density lipoprotein associated serum paraoxonase-1 (PON1) is essential for PON1-mediated inhibition of low density lipoprotein oxidation and stimulation of macrophage cholesterol efflux. J Biol Chem 2006;281:7657-65.

13. Ahmed Z, Babaei S, Maguire GF, et al. Paraoxonase-1 reduces monocyte chemotaxis and adhesion to endothelial cells due to oxidation of palmitoyl, linoleoyl glycerophosphorylcholine. Cardiovasc Res 2003;57:225-31.

14. Rosenblat M, Volkova N, Ward J, et al. Paraoxonase 1 (PON1) inhibits monocyte-to-macrophage differentiation. Atherosclerosis 2011;219:49-56.

15. Aharoni S, Aviram M, Fuhrman B. Paraoxonase 1 (PON1) reduces macrophage inflammatory responses. Atherosclerosis 2013;228:353-61.

16. Shih DM, Gu L, Xia YR, et al. Mice lacking serum paraoxonase are susceptible to organophosphate toxicity and atherosclerosis. Nature 1998;394:284-7.

17. Shih DM, Xia YR, Wang XP, et al. Combined serum paraoxonase knockout/apolipoprotein E knockout mice exhibit increased lipoprotein oxidation and atherosclerosis. J Biol Chem 2000;275:17527-35.

18. Tward A, Xia YR, Wang XP, et al. Decreased atherosclerotic lesion formation in human serum paraoxonase transgenic mice. Circulation 2002;106:484-90.

19. Bhattacharyya T, Nicholls SJ, Topol EJ, et al. Relationship of paraoxonase 1 (PON1) gene polymorphisms and functional activity with systemic oxidative stress and cardiovascular risk. JAMA 2008;299:1265-76.

20. Mackness B, Quarck R, Verreth W, et al. Human paraoxonase-1 overexpression inhibits atherosclerosis in a mouse model of metabolic syndrome. Arterioscler Thromb Vasc Biol 2006;26:1545-50.

21. Acton S, Rigotti A, Landschulz KT, et al. Identification of scavenger receptor SR-BI as a high density lipoprotein receptor. Science 1996;271:518-20.

22. Rigotti A, Trigatti BL, Penman M, et al. A targeted mutation in the murine gene encoding the high density lipoprotein (HDL) receptor scavenger receptor class B type I reveals its key role in HDL metabolism. Proc Natl Acad Sci U S A 1997;94:12610-5.

23. Leiva A, Verdejo H, Benítez ML, et al. Mechanisms regulating hepatic SR-BI expression and their impact on HDL metabolism. Atherosclerosis 2011;217:299-307.

24. Van Eck M, Hoekstra M, Hildebrand RB, et al. Increased oxidative stress in scavenger receptor $\mathrm{BI}$ knockout mice with dysfunctional HDL. Arterioscler Thromb Vasc Biol 2007;27:2413-9.

\section{Zhao et al. PON1 on dysfunctional HDL in Scarb1 deficient mice}

25. Cao J, Xu Y, Li F, et al. Protein markers of dysfunctional HDL in scavenger receptor class B type I deficient mice. J Transl Med 2018;16:155.

26. Zanoni P, Khetarpal SA, Larach DB, et al. Rare variant in scavenger receptor BI raises HDL cholesterol and increases risk of coronary heart disease. Science 2016;351:1166-71.

27. Xu YY, Du F, Meng B, et al. Hepatic overexpression of methionine sulfoxide reductase A reduces atherosclerosis in apolipoprotein E-deficient mice. J Lipid Res 2015;56:1891-900.

28. Zhou C, Cao J, Shang L, et al. Reduced paraoxonase 1 activity as a marker for severe coronary artery disease. Dis Markers 2013;35:97-103.

29. Xu Y, Li F, Zhao X, et al. Methionine sulfoxide reductase A attenuates atherosclerosis via repairing dysfunctional HDL in scavenger receptor class B type I deficient mice. FASEB J 2020;34:3805-19.

30. Meurs I, Hoekstra M, van Wanrooij EJ, et al. HDL cholesterol levels are an important factor for determining the lifespan of erythrocytes. Exp Hematol 2005;33:1309-19.

31. Imai $Y$, Morita $H$, Kurihara $H$, et al. Evidence for association between paraoxonase gene polymorphisms and atherosclerotic diseases. Atherosclerosis 2000;149:435-42.

32. Levy D, Reichert CO, Bydlowski SP. Paraoxonases Activities and Polymorphisms in Elderly and Old-Age Diseases: An Overview. Antioxidants (Basel) 2019;8:118.

33. Nicholls SJ, Hazen SL. Myeloperoxidase, modified lipoproteins, and atherogenesis. J Lipid Res 2009;50 Suppl:S346-51.

34. Haraguchi $Y$, Toh R, Hasokawa M, et al. Serum myeloperoxidase/paraoxonase 1 ratio as potential indicator of dysfunctional high-density lipoprotein and risk stratification in coronary artery disease. Atherosclerosis 2014;234:288-94.

35. Gaidukov L, Tawfik DS. High affinity, stability, and lactonase activity of serum paraoxonase PON1 anchored on HDL with ApoA-I. Biochemistry 2005;44:11843-54.

36. Chan GK, Witkowski A, Gantz DL, et al. Myeloperoxidase-mediated methionine oxidation promotes an amyloidogenic outcome for apolipoprotein A-I. J Biol Chem 2015;290:10958-71.

37. Ljunggren SA, Levels JH, Hovingh K, et al. Lipoprotein profiles in human heterozygote carriers of a functional mutation P297S in scavenger receptor class B1. Biochim Biophys Acta 2015;1851:1587-95.

38. Qi Y, Liu J, Wang W, et al. Apolipoprotein E-containing high-density lipoprotein (HDL) modifies the impact of 
cholesterol-overloaded HDL on incident coronary heart disease risk: A community-based cohort study. J Clin Lipidol 2018;12:89-98.e2.

39. Hatters DM, Peters-Libeu CA, Weisgraber KH. Apolipoprotein E structure: insights into function. Trends Biochem Sci 2006;31:445-54.

40. Kunnen S, Van Eck M. Lecithin:cholesterol acyltransferase: old friend or foe in atherosclerosis? J Lipid Res 2012;53:1783-99.

41. Brites F, Martin M, Guillas I, et al. Antioxidative activity of high-density lipoprotein (HDL): Mechanistic insights into potential clinical benefit. BBA Clin 2017;8:66-77.

42. Chen C, Loo G. Effect of peroxyl radicals on lecithin/ cholesterol acyltransferase activity in human plasma. Lipids 1995;30:627-31.

43. Ma K, Forte T, Otvos JD, et al. Differential additive effects of endothelial lipase and scavenger receptorclass B type I on high-density lipoprotein metabolism in knockout mouse models. Arterioscler Thromb Vasc Biol 2005;25:149-54.

44. Zannis VI, Fotakis P, Koukos G, et al. HDL biogenesis, remodeling, and catabolism. Handb Exp Pharmacol 2015;224:53-111.

Cite this article as: Zhao XJ, Liu LC, Guo C, Shen WW, Cao J, Du F, Wu DF, Yu H. Hepatic paraoxonase 1 ameliorates dysfunctional high-density lipoprotein and atherosclerosis in scavenger receptor class B type I deficient mice. Ann Transl Med 2021;9(13):1063. doi: 10.21037/atm-21-682
45. Westerterp M, Bochem AE, Yvan-Charvet L, et al. ATP-binding cassette transporters, atherosclerosis, and inflammation. Circ Res 2014;114:157-70.

46. Hafiane A, Genest J. ATP binding cassette A1 (ABCA1) mediates microparticle formation during highdensity lipoprotein (HDL) biogenesis. Atherosclerosis 2017;257:90-9.

47. Graf GA, Yu L, Li WP, et al. ABCG5 and ABCG8 are obligate heterodimers for protein trafficking and biliary cholesterol excretion. J Biol Chem 2003;278:48275-82.

48. Schulman IG. Liver X receptors link lipid metabolism and inflammation. FEBS Lett 2017;591:2978-91.

49. Malerød L, Juvet LK, Hanssen-Bauer A, et al. Oxysterolactivated LXRalpha/RXR induces hSR-BI-promoter activity in hepatoma cells and preadipocytes. Biochem Biophys Res Commun 2002;299:916-23.

50. Laffitte BA, Repa JJ, Joseph SB, et al. LXRs control lipidinducible expression of the apolipoprotein $\mathrm{E}$ gene in macrophages and adipocytes. Proc Natl Acad Sci U S A 2001;98:507-12.

51. Chang TY, Li BL, Chang CC, et al. Acyl-coenzyme A:cholesterol acyltransferases. Am J Physiol Endocrinol Metab 2009;297:E1-9. 


\section{Supplementary}

Table S1 The information of all primary antibodies and loading amount of proteins in western blot

\begin{tabular}{|c|c|c|c|c|c|}
\hline Primary antibody & Origin & Company & Cat. No. & Dilution rate & Sample loading \\
\hline APOA1 polyclonal antibody & UK & Abcam & Ab7614 & $1: 1000$ & $30 \mu \mathrm{g}$ or $0.5 \mu \mathrm{L}$ (plasma) \\
\hline APOE monoclonal antibody & UK & Abcam & Ab183597 & $1: 1000$ & $30 \mu \mathrm{g}$ or $1 \mu \mathrm{L}$ (plasma) \\
\hline LCAT monoclonal antibody & UK & Abcam & Ab109417 & $1: 1000$ & $0.5 \mu \mathrm{L}$ (plasma) \\
\hline A1AT monoclonal antibody & UK & Abcam & Ab133642 & $1: 1000$ & $1 \mu \mathrm{L}$ (plasma) \\
\hline APOA4 polyclonal antibody & USA & R\&D Systems & AF8125 & $1: 1000$ & $1 \mu \mathrm{L}$ (plasma) \\
\hline LDLR monoclonal antibody & UK & Abcam & Ab52818 & $1: 500$ & $40 \mu \mathrm{g}$ \\
\hline LXR $\alpha$ polyclonal antibody & UK & Abcam & Ab28478 & $1: 1000$ & $30 \mu g$ \\
\hline ACAT polyclonal antibody & UK & Abcam & Ab93477 & $1: 1000$ & $30 \mu g$ \\
\hline $\mathrm{p}-\mathrm{NF}-\kappa \mathrm{B}$ monoclonal antibody & USA & Cell Signaling Technology & 3033 & $1: 1000$ & $50 \mu g$ \\
\hline GAPDH polyclonal antibody & USA & Santa Cruz Biotechnology & SC-25778 & $1: 1000$ & \\
\hline
\end{tabular}

A1AT, alpha 1 antitrypsin; ABCA1, ATP-binding cassette A1; ABCG8, ATP-binding cassette G8; ACAT, acyl-coenzyme A cholesterol acyltransferase; APOA1, apolipoprotein A1; APOA4, apolipoprotein A4; APOE, apolipoprotein E; GAPDH, glyceraldehyde-3-phosphate dehydrogenase; LCAT, lecithin-cholesterol acyltransferase; LDLR, LDL receptor; LXR $\alpha$, liver X receptor alpha; $p-N F-\kappa B$, phosphorylated nuclear factor kappa B; PON1, paraoxonase1; SAA, serum amyloid A. 